\title{
HÁBITOS DE ESTUDIO VS. FRACASO ACADÉMICO
}

\author{
Martha Rocío Torres Narváez \\ Fisioterapeuta y profesora de la \\ Escuela de Medicina y Ciencias de la Salud \\ Universidad del Rosario \\ Bogotá, Colombia \\ Ingrid Tolosa Guzmán \\ Fisioterapeuta y profesora de la \\ Escuela de Medicina y Ciencias de la Salud \\ Universidad del Rosario \\ Bogotá, Colombia \\ María del Carmen Urrea González \\ Fisioterapeuta de la Universidad Industrial de Santander \\ Profesora de la Universidad de la Sabana \\ Bogotá, Colombia \\ Angélica María Monsalve Robayo \\ Terapeuta ocupacional y profesora de la \\ Escuela de Medicina y Ciencias de la Salud \\ Universidad del Rosario \\ Bogotá, Colombia
}

Resumen: El objetivo del artículo es exponer algunas estrategias de apoyo pedagógico que, articuladas con la estructura curricular del Programa de Fisioterapia de la Universidad del Rosario, apoyan el proceso de aprendizaje de los estudiantes a partir de las categorías analizadas con la aplicación del instrumento "Inventario de hábitos de estudio" a los estudiantes que cursaron una de las asignaturas de mayor fracaso académico. Los hábitos de estudio tienen una implicación en el rendimiento académico y esto influye en cómo se enfrenta el reto de asumir las responsabilidades de ser universitario. Como parte de la metodología, se realizó un análisis de los resultados de la aplicación del inventario antes nombrado, en aras de identificar y replantear las estrategias pedagógicas.

\section{Introducción}

El tema de la deserción y del fracaso académico es fundamental para las instituciones de educación superior en su direccionamiento estratégico, puesto que el compromiso con la formación de profesionales implica una evaluación constante de los procesos pedagógicos que propicie la integralidad en su proyecto educativo y el ejercicio de la autonomía en la comunidad académica. Este ejercicio 
Para esto se revisaron los planes de asignaturas y se determinaron las actividades extracurriculares que se diseñaron para abordar la problemática de la deserción estudiantil relacionada con el fracaso académico. El estudio realizado determinó la importancia del desarrollo de habilidades o hábitos de estudio apropiados para el buen desempeño del estudiante universitario. Además, comprobó que deben considerarse en el entorno universitario la cultura de aprendizaje en el proceso de formación, las habilidades de trabajo en equipo, la apropiación y el desarrollo de conocimiento, así como las buenas relaciones interpersonales, para disminuir el fracaso académico y mejorar los hábitos de estudio.

Palabras clave: Fracaso académico, hábitos de estudio y estrategias pedagógicas.

\begin{abstract}
The objective of this article is to present some pedagogical strategies that, articulated with the curricular structure of the Program of Physiotherapy of the University of the Rosary, support the process of learning from the categories analyzed with the application of the instrument "Inventory of Habits of Study" to the students who attended one of the subjects of greater academic failure. The study habits have an implication in the academic performance. They influence on how the challenge to assume the responsibilities of being a college student is faced. As part of the methodology, an analysis of the results of the inventory was conducted, for the sake of identifying and reframing the pedagogical strategies. For this purpose, the course curricula were reviewed and the extracurricular activities that were designed to approach the problem of student desertion related to the academic failure were determined. The study determined the importance of the development of abilities or appropriate habits of study for the good performance of the university student. In addition, the research verified that the culture of learning in the university surroundings must be considered in the process of training, the abilities of team work, the development of knowledge, as well as the good interpersonal relationships, in order to diminish the academic failure and to improve the study habits.
\end{abstract}

Key words: Academic failure, habits of study and pedagogical strategies. que el estudiante ejerce en su proceso de aprendizaje se enriquece cuando aprende cómo organizar sus recursos para el estudio, es decir, cuando adquiere conciencia de sus hábitos de estudio y de la manera en que, a partir de estos, se acerca o se aleja de su éxito académico.

Este trabajo se propone exponer algunas estrategias de apoyo pedagógico que, articuladas con la estructura curricular del Programa de Fisioterapia de la Universidad del Rosario, Bogotá, Colombia, apoyan el proceso de aprendizaje de los estudiantes a partir de las categorías analizadas con la aplicación del instrumento "Inventario de hábitos de estudio" a los estudiantes que cursaron una de las asignaturas de mayor fracaso académico.

El programa de Fisioterapia de la Universidad del Rosario tiene un enfoque curricular centrado en el estudiante, con el cual busca que el educando desarrolle la capacidad de pensar por sí mismo, de formularse preguntas y de encontrar soluciones satisfactorias a los problemas de su profesión.

El currículo integrado promueve un modelo educativo que ofrece un continuo movimiento de elementos con diversos puntos de vista, que permiten obtener habilidades de relación (comunicativas, cognitivas y procedimentales), donde se realimentan los intereses y habilidades espirituales, cognitivas, físicas y afectivas, sin precisar jerarquías dominantes (Universidad del Rosario, Facultad de Rehabilitación y Desarrollo Humano, 2004).

Este currículo es un modelo de enseñanza que reconoce la importancia de las relaciones entre las formas de conocimiento, las actitudes y las experiencias de vida, enfocadas a la organización de procesos educativos que trascienden la mirada lineal del plan de estudios. Esta propuesta curricular enlaza diferentes aspectos de la formación, de manera asociativa y significativa, desde y hacia los objetos de estudio de los programas de pregrado, en perspectiva interdisciplinar 
y transdisciplinar (Maldonado, 2001). Considera el aprendizaje desde una visión dinámica del mundo complejo e interactivo.

El sistema de créditos como una estrategia de organización curricular y de movilidad para el estudiante propicia la cultura de la autogestión del conocimiento y facilita los mecanismos de transferencia estudiantil en el contexto nacional e internacional. Según el programa de fisioterapia, este sistema se desarrolla en forma presencial y requiere de una disponibilidad de tiempo completo por parte del estudiante, visualizándose en escenarios de trabajo extraclase y directo con el docente (Universidad del Rosario, Facultad de Rehabilitación y Desarrollo Humano, 2004).

Con el sistema de créditos se busca privilegiar la formación básica que fundamenta el ejercicio de las profesiones y disciplinas, además de abrir opciones para que los estudiantes, teniendo en cuenta sus intereses y habilidades, profundicen en áreas particulares de su profesión o elijan asignaturas de otras áreas, de acuerdo con el portafolio de oferta académica que tenga disponible la Universidad cada semestre.

La malla curricular se compone de doscientos créditos académicos para la formación del fisioterapeuta, los cuales se enmarcan en los campos de formación científico-básico, social-humanístico, especifico (profesional) e investigativo. El plan de estudios considera tres tipos de asignaturas, a saber: indispensables, complementarias y electivas, propuestas en diez semestres que se desarrollan desde un modelo pedagógico conceptual activo por medio del aprendizaje significativo.

Anteriormente, en el diseño curricular no se contemplaban asignaturas de formación en competencias básicas tales como la lectura profunda, la dialéctica, la crítica y la competencia comunicativa, las cuales fueron incluidas en la reforma curricular del plan actual correspondiente al año 2005 de formación del fisioterapeuta.
Por otra parte, en el plan de estudios algunas de las asignaturas de formación básica y profesional tienen altos índices de fracaso académico. Las características que estas tienen en común son su alta intensidad horaria presencial, la cual supera las ocho horas semanales, la complejidad de los logros perseguidos, el número de estudiantes por grupo -entre 35 y 45 estudiantes-, el número de profesores que las orientan, la dependencia de prerrequisitos para su comprensión adecuada, así como la necesidad de una apropiación responsable del proceso de aprendizaje.

Algunas de las asignaturas son denominadas indispensables, ya que capacitan al estudiante en el desarrollo de habilidades para el abordaje terapéutico del individuo en condiciones de deficiencia funcional del movimiento corporal, mediante el estudio de diferentes técnicas de tratamiento en fisioterapia, las cuales demandan la comprensión y aplicación de principios y métodos relativos a ellas. Estas materias corresponden a la formación fundamental del fisioterapeuta.

Al llegar a estas asignaturas los estudiantes han superado el $40 \%$ de la malla curricular. Este primer ciclo de formación privilegia procesos memorísticos y de repetición relacionados con categorías conceptuales propias de materias básicas. Por lo tanto, los estudiantes no logran estructurar un pensamiento categorial que les permita realizar de manera analítica, argumentativa y crítica la toma de decisiones requerida para su desempeño profesional.

El componente de la malla curricular que involucra el razonamiento clínico demanda procesos de pensamiento que van desde la organización conceptual hasta la categorización de los diferentes niveles de conocimiento, requeridos para la toma de decisiones.

Las prácticas pedagógicas que predominan en este tipo de asignaturas (indispensables y complementarias) son la mesa redonda, el club de revista, el taller, resúmenes analíticos de investigación, actividades colaborativas, las demostraciones prácticas y el estudio de caso. Todas fundamentadas en un modelo pedagógico 
conceptual y encaminado al aprendizaje significativo.

El aprendizaje significativo propone tres habilidades en el aprendizaje del estudiante: asociar contenidos previos, percibir la necesidad de vinculo entre aprendizajes y disponerse a correlacionar los dos anteriores (Ardila y Tolosa, 2006).

El material de enseñanza empleado comprende la guía de estudio, la guía didáctica, bibliografía referida, artículos de revistas científicas, bases de datos, recursos bibliográficos electrónicos y portafolio. Estos componentes actúan como mediadores del proceso de aprendizaje. En cuanto a las estrategias de evaluación, se incluyen pruebas escritas, orales de tipo práctico y sustentación de casos.

La estrategia implementada para abordar la problemática relacionada con el fracaso académico incluyó un análisis del desempeño en el periodo comprendido entre los años 2005 y 2006. A partir de ese año se ha realizado un seguimiento tutorial y se han instaurado asesorías y monitorias académicas.

El alto índice de fracaso académico en las asignaturas tanto indispensables como complementarias es constante a pesar de las actividades realizadas; por ende, se ha motivado el análisis de los hábitos de estudio y su relación con el desarrollo de habilidades cognitivas que son estimuladas mediante las estrategias pedagógicas implementadas en el programa.

Es así como la aplicación del "Inventario de hábitos de estudio" (Pozar, 2002) en algunos de los estudiantes que inscribieron una las asignaturas indispensables de alto fracaso académico evidenció en sus resultados que tanto la manera como los factores ambientales, la planificación del estudio, la utilización de materiales y la asimilación de contenidos confluyen en el desempeño académico de los estudiantes.

El Inventario de Pozar (2002) es una prueba elaborada con el propósito de detectar hasta qué punto el estudiante conoce su oficio. Consta de 90 elementos organizados en once factores, distribuidos por escalas, de la siguiente manera:

Escala I. Condiciones ambientales de estudio: cuenta con 18 elementos que recopilan los condicionamientos del ambiente que rodean al estudiante, como son los personales, el ambiente físico, el comportamiento académico y el rendimiento.

Escala II. Son doce elementos que indagan sobre la planificación del estudio; esta incluye horarios de todas las actividades, además de contemplar espacios de descanso, la organización de los materiales y elementos necesarios para el estudio.

Escala III. Es la utilización de materiales que, por medio de 15 elementos, establece el manejo de los libros, las lecturas y los resúmenes.

Escala IV. Asimilación de contenidos: consta de dos factores y 15 elementos para determinar tanto el grado de memorización como la personalización que incluye el trabajo personal y en equipo.

La estructura del Inventario permite correlacionar las estrategias pedagógicas con elementos para el aprendizaje tales como actividades de planificación, organización y utilización de materiales de estudio, en las cuales es fundamental la disposición y el compromiso del estudiante con el aprendizaje.

\section{Estrategias pedagógicas y habilidades cognitivas}

La formación de profesionales en Fisioterapia, por la naturaleza de su objeto de estudio y por las implicaciones que tiene su desempeño profesional en la calidad de vida de las personas, demanda unas condiciones especiales dirigidas a asegurar una eficiente prestación de servicios en 
salud. Estas características especiales están dadas por el componente de aprendizaje que está implícito en la interacción terapéutica en todos y cada uno de los escenarios de acción y niveles de atención en salud.

Con base en lo anterior, el proyecto educativo debe considerar estrategias pedagógicas que afiancen la adquisición de habilidades cognitivas, procedimentales y actitudinales que se reflejen en el ejercicio profesional responsable de sus egresados. Es decir, una cultura de aprendizaje en los procesos, habilidades de trabajo en equipo, apropiación y desarrollo de conocimiento, así como buenas relaciones interpersonales.

Apropiarse de las estrategias pedagógicas que aseguren el aprendizaje de los estudiantes es un reto para los profesores universitarios. La ideación e implementación de estas estrategias ameritan del análisis no solo procedimental del desarrollo de una metodología determinada, sino de la influencia del pensamiento en el proceso de adquisición y uso útil del conocimiento adquirido (Jensen, 2004).

Ese pensamiento regulado por el cerebro requiere procesar información que proviene del exterior y considerar la anticipación para favorecer el recuerdo de situaciones, lo que permite la comparación y la escogencia de datos relevantes en los que se focaliza la atención, a fin de elaborar un análisis y un reconocimiento de lo seleccionado para organizar ese conocimiento adquirido y traducirlo en aprendizajes significativos.

En este sentido, el proceso demanda de la iniciativa del estudiante para explorar, leer, averiguar, curiosear sobre un "algo" remoto que posteriormente constituye un motivo específico, bajo unos parámetros y redes de circuitos cerebrales que revisan permanentemente la información procesada.

Entonces, el aprendizaje significativo se comporta como aquel que crea nuevas redes y sincronías por medio de la creación, actualización y producción del saber, lo que implica el uso inteligente de la información (Obaya, García, Noé, Noé, Tapia y Hernández, 1996).

\section{Metodología}

Dado el interés del Programa de Fisioterapia por disminuir el fracaso académico de los estudiantes en las asignaturas indispensables, desde la coordinación académica se realizó un análisis de los resultados de la aplicación del inventario antes mencionado en aras de fundamentar la pertinencia de replantear las estrategias pedagógicas hasta la fecha utilizadas en el Programa. Esto permitirá consolidar el cumplimiento de los objetivos de formación de este en cada uno de los ciclos de la malla curricular e incidirá positivamente en el desempeño académico.

Para ello se identificaron las estrategias pedagógicas que empleaban los profesores del Programa y que se encontraban registradas en los planes de asignaturas. De igual manera se determinaron las actividades extracurriculares que se diseñaron para abordar la problemática de la deserción estudiantil relacionada con el fracaso académico.

Otro factor considerado en este análisis corresponde a la manera en que los estudiantes adecuan las condiciones ambientales, planifican su estudio, organizan y emplean materiales de aprendizaje en relación con las metas que establecen en su proceso de formación profesional. En esta parte se consideró la información referida en la aplicación del "Inventario de hábitos de estudio" de Pozar (2002), que se aplicó a los estudiantes en el año 2006.

\section{Resultados}

Los planes de asignatura reportaron las actividades pedagógicas que los profesores diseñan y establecen para que 
los estudiantes mejoren su participación en clase y se favorezca el desarrollo de la misma, dado que dentro del modelo curricular el estudiante debe preparar anticipadamente la temática a desarrollar en la sesión presencial de la asignatura y asumir tiempo extraclase para trabajar en los logros y objetivos que debe ir alcanzando en el proceso de formación.

En estos se destacan las referencias que hacen al empleo de tecnologías de información y comunicación; por ejemplo, la plataforma Babel, que soporta en gran medida la puesta en marcha del modelo pedagógico. Asimismo, actividades como talleres, estudio de casos, exposiciones, desarrollo de guías de estudio y didácticas, clase magistral, presentación de trabajos de investigación, controles de lectura y clubes de revista. Todo esto conjugado con mecanismos de retroalimentación permanente y demostraciones prácticas constituye las herramientas actuales para promover el aprendizaje significativo.

El programa, bajo lineamientos institucionales, desarrolla acciones integradas para el abordaje de la problemática de la deserción estudiantil. El estudio de deserción realizado por la Facultad entre los años 2001-2003 reveló que la mayor causa de deserción es académica (79\%) y que el $21 \%$ es por situaciones no académicas, dentro de las que se destacan problemas familiares, dificultades económicas, de seguridad y embarazos (Universidad del Rosario, Facultad de Rehabilitación y Desarrollo Humano, Programa de Fisioterapia, 2006).

Los programas de tutorías, asesorías y monitorías académicas apoyan el proceso de aprendizaje de la población estudiantil para acompañar la apropiación del enfoque curricular y el modelo pedagógico que deben hacer los estudiantes como actores del proyecto educativo. Aunque se ha destinado tiempo para la ejecución de estas acciones, aún no se cuenta con la medición del impacto que genera en los procesos académicos del programa.
La aplicación del "Inventario de Hábitos de Estudio" (Gráfico 1) realizada a Toma de decisiones IV, una de las asignaturas indispensables de mayor fracaso académico, reportó que el 33\% de los estudiantes encuentran que los factores ambientales de estudio no son satisfactorios para el proceso de aprendizaje. El 41\% manifiesta que la planificación del estudio es insuficiente, el $61 \%$ de los estudiantes valora la utilización de materiales como un factor positivo, el 93\% evalúa positivamente la asimilación de contenidos, lo cual invita a validar el sentido y significado que tiene para los estudiantes y profesores este elemento en una asignatura teóricopráctica que demanda la argumentación y toma de decisiones de propuestas terapéuticas en el ámbito de las deficiencias del movimiento corporal humano. Se hace necesario implementar estrategias que fortalezcan los hábitos en los estudiantes a fin de conseguir el éxito académico y de formación personal considerando las condiciones particulares de cada grupo de estudiantes (Torres, Tolosa, Urrea y Monsalve, 2009).

\section{Conclusión y discusión}

Los estudios realizados señalan la importancia del desarrollo de habilidades o hábitos de estudio apropiados para el buen desempeño del estudiante universitario. Para ilustrar, se refiere a lo reportado por Grajales (2002), quien estudiando la conveniencia de hacer esfuerzos dirigidos a mejorar las habilidades de estudio, encontró que los estudiantes que participaron en un programa de formación de hábitos denominado "ExCel" obtuvieron mejores resultados en el desarrollo de sus hábitos de estudio, comparados con otros que no participaron.

Asimismo, se sugiere en este estudio que los estudiantes que están ingresando a los programas universitarios necesitan 


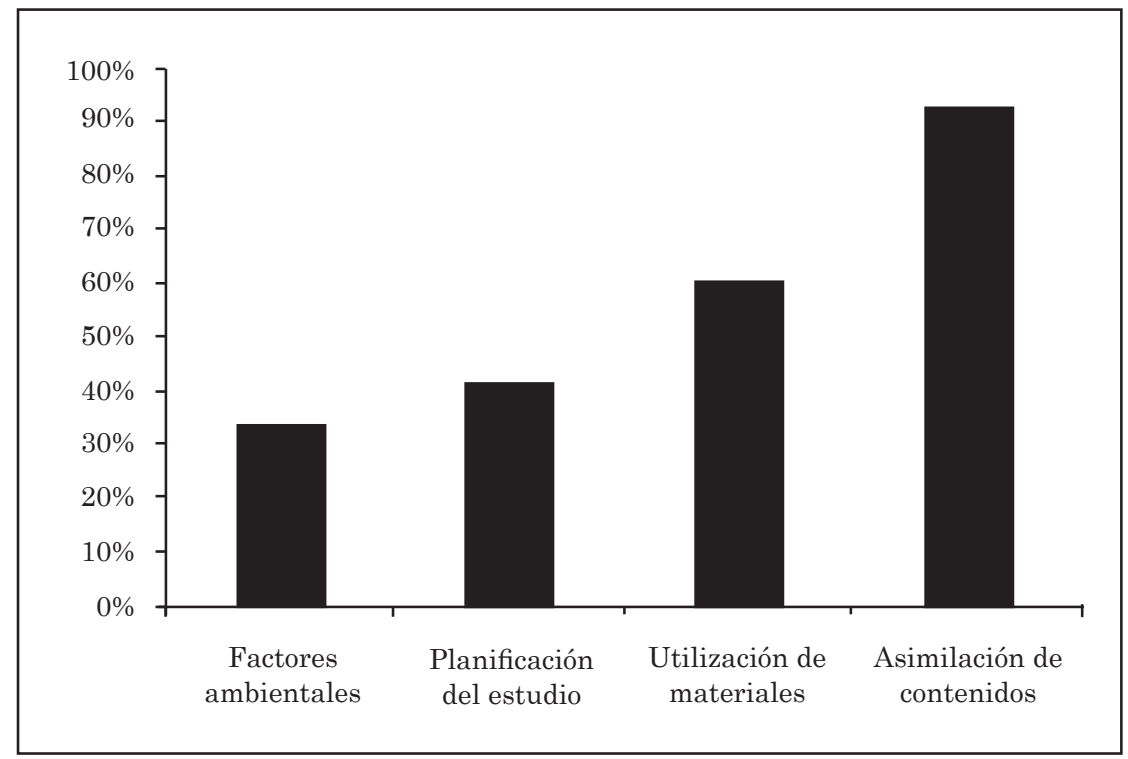

Fuente: Elaboración propia.

apoyo y una clara comprensión de los medios que pueden utilizar para lograr el éxito, y recomienda que los maestros animen a los estudiantes a probar diferentes estrategias, desarrollar una variedad de herramientas útiles y descubrir qué es lo que mejor les funciona tanto para su aprendizaje como para lograr las metas de su vida.

El sistema de créditos demanda responsabilidad y compromiso del estudiante y del profesor para lograr la meta de aprendizaje, puesto que involucra en gran medida un componente de trabajo independiente que debe ser planeado por el estudiante y dirigido por el profesor. Es aquí donde los hábitos de estudio constituyen un eje integrador y facilitador del proceso de aprendizaje por cuanto activan los recursos cognitivos, ambientales y procedimentales con los que cuenta el estudiante.

El alto porcentaje en la asimilación de contenidos reportada en la aplicación del
Inventario invita a validar este elemento para estudiantes y profesores en una asignatura indispensable, la cual demanda la argumentación y toma de decisiones de propuestas terapéuticas en el ámbito de las deficiencias del movimiento corporal humano. Esto más aún cuando hasta el momento el estudiante no ha tenido experiencias integradoras que le permitan estructurar el pensamiento categorial y argumentativo que demanda este tipo de asignaturas dentro de su proceso de formación.

En otro estudio, Connelly, DuBois y Staley (1998) proponen las estrategias como actos que permiten mantener y propiciar la salud, tanto física como mental. Estas estrategias transferidas al estudio favorecen la adquisición de conocimiento y la creación de hábitos, lo que involucra la integración de la voluntad, la motivación y el aislamiento psicológico. Adicionalmente, se define la capacidad de estudio como las acciones mentales que 
implican observación, asociación y síntesis para mejorar el aprendizaje.

Una investigación sobre hábitos realizada por Téllez-Godoy (2005) encuentra resultados poco satisfactorios en las variables de estudio, debido a que los estudiantes participantes en su trabajo de investigación informaron que hacen poco ejercicio, pocas veces desayunan antes de ir a estudiar, no consumen los alimentos a la misma hora, no duermen las ocho horas diarias, no tienen un lugar fijo para estudiar y pocas veces preguntan cuando no entienden.

Asimismo, esto se ve ligado a los perfiles individuales provenientes de las aptitudes, actitudes y estrategias derivadas de las experiencias educativas y personales que hacen que tanto las capacidades cognitivas y metacognitivas asociadas a la juventud y madurez (tales como razonamiento abstracto y descontextualizado, razonamiento hipotético y razonamiento deductivo), así como a los intereses vocacionales derivados de las preferencias o aptitudes específicas y no siempre bien encauzados supongan fuentes importantes de diversidad individual (Escalante, Escalante, Linzaga y Merlos, 2008).

Estos mismos autores hacen alusión a la importancia de las características vinculadas al ritmo de aprendizaje, al nivel de conocimientos de entrada, a las estrategias de aprendizaje, a la dedicación, esfuerzo o implicación en las tareas y a la responsabilidad para alcanzar los objetivos propuestos en la tarea formativa.

Si los elementos anteriores son importantes para analizar los hábitos de estudio, existen también factores que influyen en los procesos de adquisición de aprendizajes y utilización efectiva del conocimiento. Estos factores se relacionan con las habilidades cognitivas, ya que constituyen una de las bases para determinar el tipo de conocimiento que se desea impartir (declarativo o procedimental), el tipo de dominio (cuantitativo, técnico o verbal) con el que se relacionan las diferentes habilidades y la complejidad de las tareas asignadas. En este caso, la ejercitación, la práctica y la automatización de las destrezas son importantes para los estudiantes, especialmente para aquellos con dificultades de aprendizaje o bajas aptitudes (García, Asensio, Carballo, García y Guardia, 2005).

De la misma manera, en la relación cognición-rendimiento interfiere el tipo de entorno instructivo y, más concretamente, el método educativo que se utilice. Esto debido a que indirectamente exige al estudiante diferentes destrezas, sobre todo relacionadas con el control de su aprendizaje.

Un elemento de análisis dentro de estos estudios y la discusión que aquí se presenta es el que compete a los estilos cognitivos definidos por Witkin (citado por Schmeck, 1988, p. 5) como "la forma típica de procesar información, independientemente de si la información tiene su fuente primaria en el mundo exterior o en nosotros, y cuando es del mundo exterior, indiferentemente si proviene de las cosas o de las personas y sus actividades". Lo que lleva a explicar que el ser humano utiliza diferentes acciones mentales como percibir, reflexionar, atender, recordar, categorizar y conceptuar desde lo netamente cognitivo, sin olvidar que los rasgos y características socio-emocionales tienen también una influencia significativa en el proceso de aprendizaje (Messick, 1984).

Entonces, cuando se afirma que estos estilos cognitivos influyen e impactan los proyectos educativos porque debe pensarse en ellos en función de los sujetos que los viven, surgen las estrategias pedagógicas que, según el programa académico que forma profesionales en salud, implican el desarrollo del razonamiento clínico. De modo que el estudiante debe construir modelos de aprendizaje mediante experiencias que integren conocimientos previos, estructuras mentales y actitudes que le permitan una toma de decisiones crítica y argumentada. 
Los modelos de aprendizaje constituyen las didácticas que, en coherencia con el enfoque curricular integrado, están centradas en el estudiante y se reconocen como mapas mentales, mapas conceptuales, mentefactos, modelos categoriales, exposiciones problema, aprendizaje colaborativo, métodos de caso, aprendizaje por descubrimiento y aprendizaje basado en problemas y en proyectos (Castaño, 2004).

El aprendizaje basado en problemas es una estrategia utilizada con frecuencia en el programa de Fisioterapia, se centra en el estudiante y permite la estructuración del conocimiento por medio del proceso de resolución de problemas, basado en la colaboración e integración en el contexto de un pequeño grupo, en el que el educando desarrolla la capacidad de trabajar en conjunto para identificar y analizar problemas y/o generar soluciones, bajo la guía de un tutor (Morales y Fitzgeral, 2004).

La simulación es otra estrategia de aprendizaje que requiere centrarse en el paciente y en el contacto temprano con este. Esta utiliza los ámbitos de simulación que imitan algún aspecto de la realidad y se establecen, en ese ambiente, situaciones similares a las que él deberá enfrentar con individuos sanos o enfermos de forma independiente, durante los diferentes momentos de su formación.

Dentro de las estrategias existentes también se encuentran las tecnologías de información y comunicación en educación superior, las cuales requieren otra serie de habilidades por parte de los estudiantes y retos para el profesor, como: capacitación más allá del área disciplinar, nuevas competencias en comunicación, uso adecuado de nuevas tecnologías en educación, incorporación de nuevas estrategias de enseñanza y aprendizaje, mayor disponibilidad de tiempo para el estudiante, facilitar la autonomía de este, estimular la participación en su formación y el uso de nuevos métodos de evaluación (Osorio, Aldana y Carvajal, 2008).

\section{Referencias bibliográficas}

Ardila, A., y Tolosa, I. (2006). Reflexiones sobre currículo y prácticas pedagógicas de los programas de rehabilitación de la Universidad del Rosario. Revista Ocupación Humana, 11(3 y 4), 74. Asociación Colombiana de Terapia Ocupacional, BogotáColombia.

Castaño, G. (2004). Independencia de los estilos de aprendizaje, de las variables cognitivas y afectivo-motivacionales. Tesis de doctorado sin publicar, Universidad Complutense de Madrid, España.

Connelly, K., DuBois, N., y Staley, R. (1998). Structured interview study of the longterm effects of a college study skills course: Traces and self-report measures [Estudio de una entrevista estructurada de los efectos a largo plazo de las habilidades de estudio de un colegio. Rastros y medidas de autoreporte]. En Asociación Americana de Investigación Educativa, Memoria del Encuentro anual de la Asociación Americana de Investigación Educativa 1998 (pp. 3-10). San Diego, CA: el autor.

Escalante, L., Escalante, Y., Linzaga, C., y Merlos, M. (2008). Comportamiento de los estudiantes en función a sus hábitos de estudio. Revista Electrónica Actualidades Investigativas en Educación, 8(2), 1-15, Instituto de Investigación en Educación, Universidad de Costa Rica, San José, Costa Rica.

Obaya, A., García, A., Noé, M., Noé, P., Tapia, V., y Hernández, V. (1996). Diagnóstico de los hábitos de estudio en alumnos de las carreras del área química. Revista Educación Química en Línea, 7(3). Consultado el 15 
de junio de 2009, de: https://www. educacionquimica.info/busqueda.php

García, N., Asensio, I., Carballo, R., García, M., y Guardia, S. (2005). La tutoría universitaria ante el proceso de armonización europea. Revista de Educación, No. 337, 189-210, Madrid, España.

Grajales, T. (2002). Hábitos de estudio de estudiantes universitarios. Informe de investigación del Centro de Investigación Educativa, Universidad Montemorelos, México.

Jensen, E. (2004). Cerebro y aprendizaje. Competencias e implicaciones educativas. Madrid: Narcea.

Maldonado, M. (2001). Las competencias, una opción de vida. Metodología para el diseño curricular. Colección Textos Universitarios. Bogotá: Ecoediciones.

Messick, S. (1984). The nature of cognitive styles: Problems and promise in educational practice [La naturaleza de los estilos cognitivos: Problemas y promesas en la práctica educativa]. Educational Psychologist, 19(2), 59-74

Morales, P., y Fitzgerald, V. (2004). Aprendizaje basado en problemas. Theoria, 13, 145-157, Universidad del Bío-Bío, Chillán, Chile.

Osorio, L., Aldana, M., y Carvajal, D. (2008). Incorporación de las TIC en educación superior: Experiencia institucional Universidad de los Andes. Ficha técnica de un trabajo para concurso del premio de experiencias en informática educativa para instituciones educativas.
Consultado de Universidad de los Andes, el 8 de junio del 2009, de: http:// www.colombiaaprende.edu.co/html/ mediateca/1607/articles-108656_ archivo.pdf

Pozar, F. (2002). Inventario de hábitos de estudio. Manual (9a. ed.). Madrid: Publicaciones de Psicología Aplicada, TEA Ediciones, S. A.

Schmeck, R. (1988). An introduction to strategies and styles of learning [Una introducción a las estrategias $y$ estilos de aprendizaje]. New York: Plenum Press.

Téllez-Godoy, L. (2005). Hábitos de estudio de los alumnos de la carrera de Ingeniero Agrónomo Fitotecnista del CEP-C SAEGRO. Tesis sin publicar presentada para optar por el grado de licenciatura, Colegio Superior Agropecuario del Estado de Guerrero, México.

Torres, M., Tolosa, I., Urrea, M., y Monsalve, A. (2009). Inventario de hábitos de estudio de estudiantes de fisioterapia en una clase para toma de decisiones. Manuscrito sin publicar, Universidad del Rosario, Bogotá, Colombia.

Universidad del Rosario, Facultad de Rehabilitación y Desarrollo Humano. (2004). Programa de Fisioterapia. Reforma curricular. Anexo 4 enfoque curricular. Bogotá, Colombia. Manuscrito sin publicar.

Universidad del Rosario, Facultad de Rehabilitación y Desarrollo Humano. (2006). Informe de autoevaluación del programa de fisioterapia con fines de renovación de la acreditación. Bogotá, Colombia. Manuscrito sin publicar. 